\title{
Aortic insufficiency following transcatheter aortic valve replacement is underestimated by echocardiography compared with cardiac MRI
}

\author{
Wissam M Abdallah ${ }^{1 *}$, Chris A Semder ${ }^{1}$, Evan L Brittain ${ }^{1}$, Michael T Baker ${ }^{1}$, Lisa A Mendes', Marshall H Crenshaw ${ }^{1}$, \\ Joseph L Fredi', Mark A Robbins ${ }^{1}$, Sonia L Scalf', William S Bradham', Sean G Hughes ${ }^{1}$, Mark A Lawson', \\ David X Zhao ${ }^{2}$
}

From 17th Annual SCMR Scientific Sessions

New Orleans, LA, USA. 16-19 January 2014

\section{Background}

The degree of aortic insufficiency (AI) after transcatheter aortic valve replacement (TAVR) has been identified as a predictor of increased mortality. Since even mild AI is associated with increased mortality in some studies, accurate quantification of post-TAVR AI is critical. Assessment of AI by echocardiography is typically performed by visual inspection and semi-quantitative methods. Most post-TAVR AI is paravalvular, however echocardiography has limited ability to quantify multiple eccentric paravalvular jets. Using flow quantification methods, cardiac MRI (CMR) may more accurately quantify AI severity post-TAVR and therefore more accurately assess risk in this population.

\section{Methods}

Twenty-three patients who underwent TAVR with a SAPIEN prosthesis were studied. All patients underwent an intraoperative transesophageal echocardiogram (TEE), as well as a post-procedure transthoracic echocardiogram (TTE) and CMR. Paravalvular AI by TTE and TEE was graded using color Doppler by quantifying the circumferential extent of AI as a percentage of the aortic annulus (none $<1 \%$, trace $1-5 \%$, mild $6-10 \%$, moderate $11-30 \%$, severe $>30 \%$ ) following recommendations from the Valve Academic Research Consortium. AI severity by CMR was quantified as the regurgitant fraction of forward aortic flow based on previously published recommendations (none $<1 \%$, trace $1-5 \%$, mild $6-15 \%$, moderate $16-48 \%$, severe $>48 \%$ ).

\section{Results}

The mean age was $79+/-10$ years; $52 \%$ were men. TTE and CMR were performed at 1 [1-1] and 4 [1-4] days postTAVR respectively (median [IQR]). The left ventricular ejection fraction (LVEF) by CMR was $65+/-10 \%$. AI severity by TTE was none in $9(39.1 \%)$, trace in $11(47.8 \%)$, and mild in 3 (13\%) patients. TEE identified trace central AI in 6 patients (26\%). Paravalvular AI by TEE was none in $4(17.4 \%)$, trace in $14(60.9 \%)$, and mild in $5(21.7 \%)$ patients. AI by CMR was none in $2(8.7 \%)$, trace in $5(21.7 \%)$, mild in 13 (56.5\%), and moderate in 3 (13\%) patients; (Figure 1). A higher proportion of patients with mild or greater AI was identified by CMR $(16 / 23,70 \%)$ compared to TTE $(3 / 23$, $13 \%$ ) and TEE (5/23, 22\%); (Figure 2).

\section{Conclusions}

CMR can reliably quantify post-TAVR AI. Echocardiography underestimates the severity of post-TAVR AI compared with CMR. CMR could be selectively utilized in patients with mild post-TAVR AI by echocardiography to screen for more significant AI. Further studies are needed to determine 1) whether echocardiography alone underestimates the risk of adverse outcomes related to postTAVR AI, and 2) whether similar degrees of post-TAVR AI by CMR also translate into adverse outcomes. 


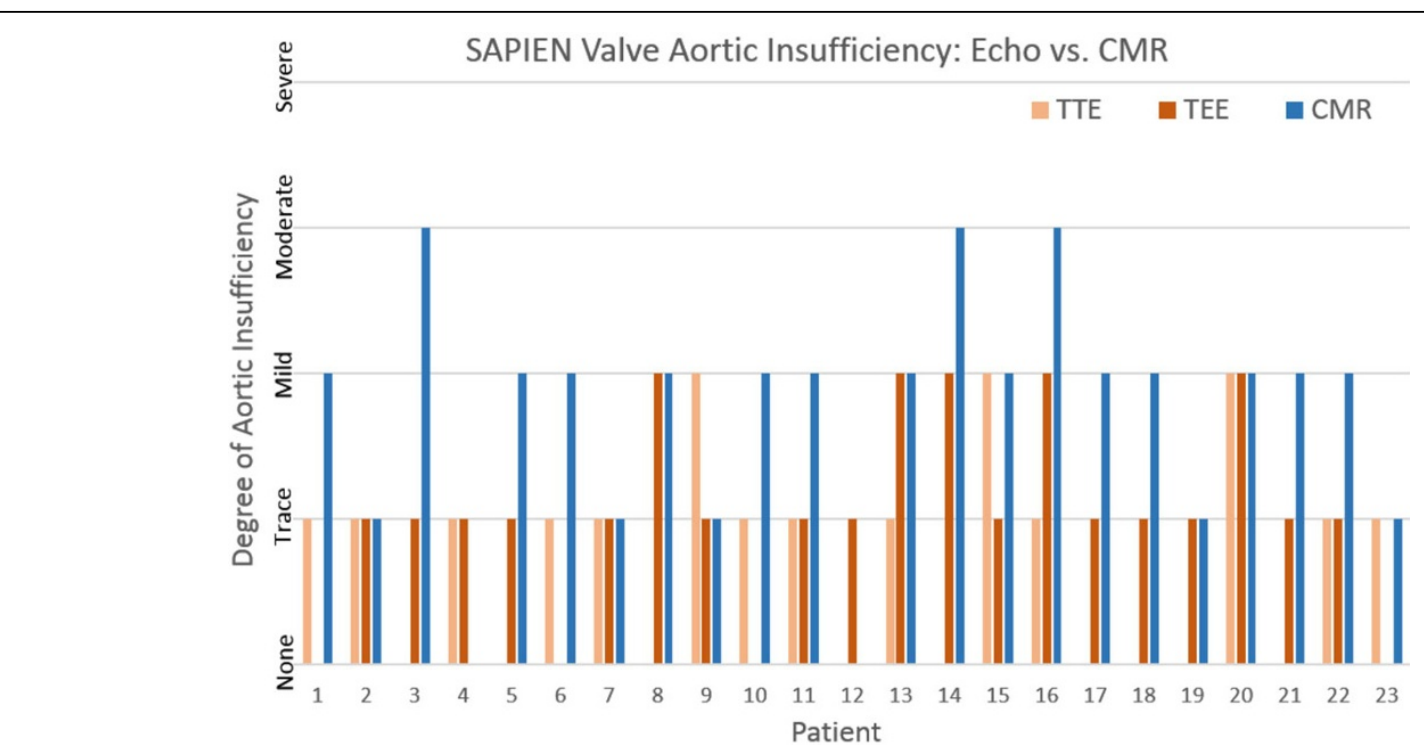

Figure 1

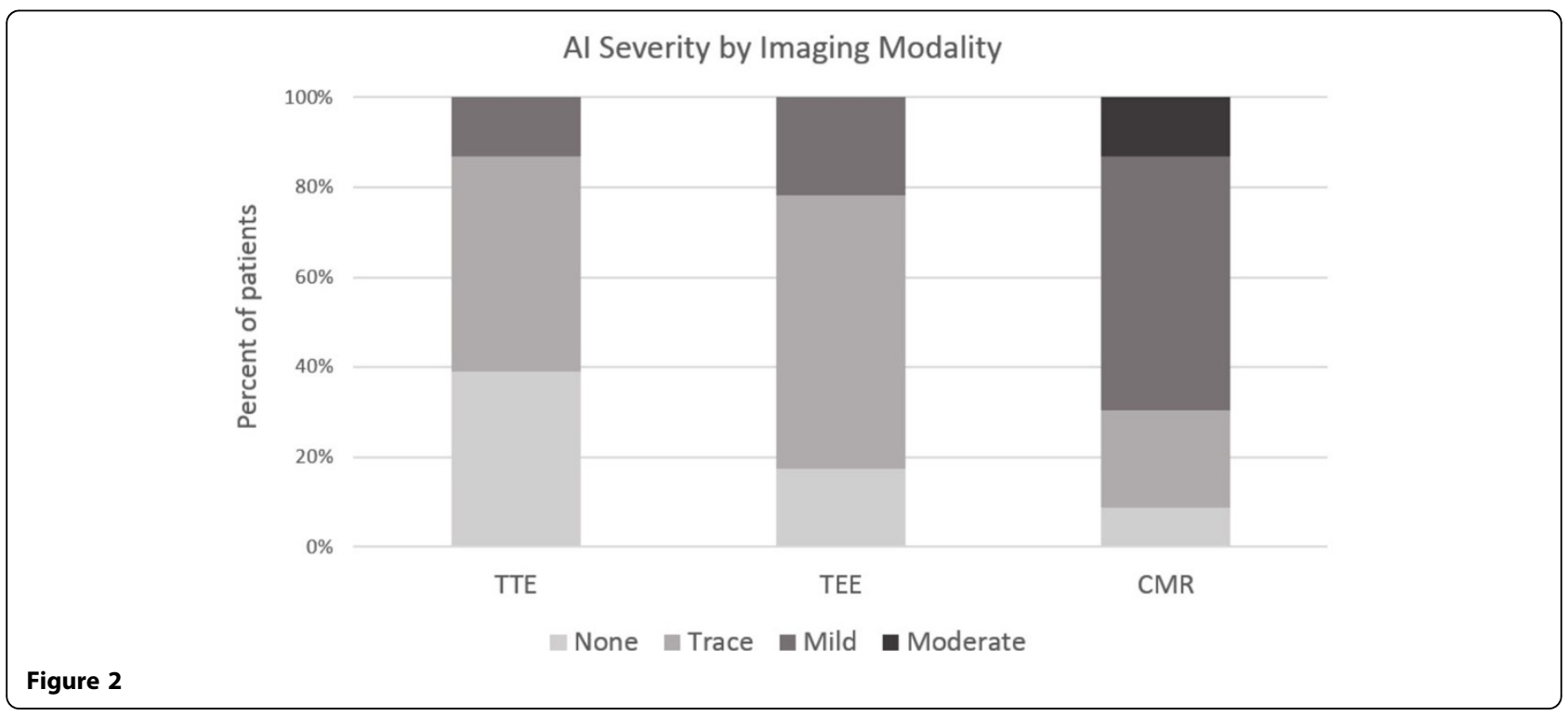

\section{Funding}

None.

\section{Authors' details}

'Division of Cardiovascular Medicine, Vanderbilt University Medical Center, Nashville, Tennessee, USA. ${ }^{2}$ Division of Cardiology, Wake Forest University, Winston-Salem, North Carolina, USA.

Published: 16 January 2014
doi:10.1186/1532-429X-16-S1-0101

Cite this article as: Abdallah et al:: Aortic insufficiency following transcatheter aortic valve replacement is underestimated by

echocardiography compared with cardiac MRI. Journal of Cardiovascular Magnetic Resonance 2014 16(Suppl 1):0101. 